\title{
SUDDEN CARDIAC DEATH: ROLE OF LEFT VENTRICULAR DYSFUNCTION
}

\author{
Bertram Pitt \\ Division of Cardiology \\ Department of Internal Medicine \\ University of Michigan Medical Center \\ Ann Arbor, Michigan 48109
}

Early studies from the Coronary Drug Project of sudden cardiac death in patients surviving an episode of myocardial infarction called attention to the importance of ventricular ectopic beats. ${ }^{1}$ In that study, complex ventricular ectopic beats recorded on a 12-hour Holter electrocardiogram were found to be associated with an excessive risk of sudden cardiac death. When the relationship was examined between complex ventricular ectopic beats and other risk factors, such as age, number of prior infarctions, angina pectoris, hypertension, serum cholesterol, glucose intolerance, cardiomegaly seen on chest X-ray films, and cigarette-smoking, complex ventricular ectopic beats were found to be independent risk factors with respect to sudden cardiac death. The importance of ventricular ectopic beats as a predictor of sudden cardiac death during the convalescent or late hospital phase of acute myocardial infarction in this and other studies has led to the use of prophylactic antiarrhythmic therapy in an attempt to prevent or reduce these ectopic beats and hence to prevent sudden cardiac death.

In a subsequent study by Schulze et al. patients surviving an episode of acute myocardial infarction underwent 24-hour Holter electrocardiographic recording and gated radionuclide left ventricular angiography prior to discharge from the hospital. ${ }^{2}$ Several important findings emerged from this study: It was noted that 26 of $29(90 \%)$ patients with complex ventricular arrhythmias (Lown Class III-V) on Holter electrocardiographic recording had a left ventricular ejection fraction of $<40 \%$ compared with only 19 of 52 (37\%) without complex ventricular arrhythmias. During a mean follow-up period of 7 months, eight patients had documented ventricular fibrillation. All eight patients who suffered sudden death were in the subset of patients with complex late hospital phase ventricular arrhythmias and a left ventricular ejection fraction of $<40 \%$. This study was one of the first to call attention to the role of ventricular dysfunction in patients with sudden cardiac death after an episode of acute myocardial infarction. The failure of left ventricular dysfunction to be recognized as an important predictor of sudden death after myocardial infarction in the Coronary Drug Project and in other previous studies may be related to the use of the cardiothoracic (CT) ratio on chest X-ray film as an index of left ventricular function. Subsequent studies have pointed out that the CT ratio is a poor index of left ventricular function and that the correlation between the $\mathrm{CT}$ ratio and left ventricular ejection fraction is poor. Schulze et al. also found that the left ventricular ejection fraction and the percent of left ventricular akinesia on gated blood pool imaging were significantly better predictors of subsequent sudden cardiac death than was peak creatine phosphokinase (CPK) determined during the episode of acute infarction. Patients who subsequently died suddenly had 
a higher peak CPK than those who survived, but this difference was not significant. In contrast, those who died suddenly had a significantly lower left ventricular ejection fraction and a greater percentage of left ventricular akinesia than those who survived. The failure of peak CPK to adequately predict those at risk for subsequent sudden death is due to the fact that it is the total myocardial damage that appears to be of importance rather than just the acute damage, which is reflected by the rise in CPK. Left ventricular ejection fraction and the percent of left ventricular akinesia reflect total myocardial damage due to previous infarction as well as acute infarction and therefore appear to be better predictors.

The importance of ventricular dysfunction as a predictor of subsequent sudden cardiac death after myocardial infarction can also be seen in a study by Ruberman et $a .^{3}$ In that study of 1739 patients with prior myocardial infarction who underwent a 1-hour electrocardiographic recording and were followed for a mean of 24.4 months, complex ventricular ectopic beats were again found to be an important predictor of subsequent sudden and nonsudden cardiac death. However, the combination of complex ventricular ectopic activity on the 1-hour electrocardiographic recording and a history of congestive heart failure showed the highest relative risk for subsequent death, both sudden and nonsudden. ${ }^{3}$

In subsequent studies by Schulze et al. it was noted that patients with complex late hospital phase ventricular ectopic beats on 24-hour Holter electrocardiographic recording prior to discharge from the hospital after an episode of acute myocardial infarction had a significantly greater incidence of multivessel coronary artery disease than did those without complex ventricular arrhythmias, as well as a significantly greater incidence of abnormally contracting myocardial segments and a lower left ventricular ejection fraction on contrast left ventriculography. ${ }^{4}$ The more extensive coronary artery disease and left ventricular dysfunction and hence increased risk of myocardial ischemia in patients with complex ventricular arthythmias after myocardial infarction were thought to help explain the relative refractoriness of many of the arrhythmias in these patients to standard antiarrhythmic therapy.

In a study of 106 patients surviving an episode of acute myocardial infarction who underwent 24-hour Holter electrocardiographic recording and coronary angiography prior to hospital dischage, Taylor et al., using univariate analysis, also noted that a low left ventricular ejection fraction, proximal left anterior descending coronary artery disease, and significant triple-vessel disease were associated with a high risk of subsequent sudden cardiac death. ${ }^{5}$ Multivariate analysis of $\mathbf{3 0}$ clinical and laboratory values identified previous myocardial infarction and a left ventricular ejection fraction of $<40 \%$ as the best predictor of mortality. In this analysis the presence of complex late hospital phase ventricular arrhythmias did not provide additional prognostic information as to subsequent sudden death once the information as to previous infarction and ejection fraction was considered. In a recent study in which 10-hour Holter electrocardiographic recordings were made on 289 survivors of an episode of acute myocardial infarction Klieger et al. also found that the occurrence of ventricular runs on electrocardiographic recording was related to left ventricular dysfunction. ${ }^{6}$ However, analysis of the data led the authors to conclude that the presence of ventricular runs on electrocardiographic recording was a marker of severe cardiac disease rather than an independent risk factor for predicting subsequent sudden cardiac death. 
On the basis of these and other studies, it appears that complex ventricular arrhythmias and left ventricular dysfunction are important predictors of death after myocardial infarction, both sudden and nonsudden. The complex ventricular arrhythmias seen during the late hospital phase of acute myocardial infarction, however, may be "innocent bystanders" and merely markers of left ventricular dysfunction rather than independent risk factors for subsequent sudden cardiac death. Indirect support for this hypothesis can be found from an analysis of several recent therapeutic trials. Attempts to reduce sudden cardiac death by prophylactic antiarrhythmic therapy or by suppressing complex ventricular ectopic activity after myocardial infarction have not been successful. For example, in a recent study of mexiletine in the postinfarction period it was found that complex ventricular ectopic beats could be successfully suppressed. ${ }^{7}$ Nevertheless, the incidence of sudden cardiac death was not reduced. In fact, there was a slightly higher incidence of death in those receiving mexiletine. Previous and ongoing trials of antiarrhythmic agents given to prevent sudden cardiac death after myocardial infarction have also failed, and although one could argue that such failure to reduce the incidence of sudden cardiac death after myocardial infarction may be due to the inadequacies of either the trial design or of currently available antiarrhythmic agents, it could equally be argued that the concept of prophylactic or suppressive antiarrhythmic therapy to prevent sudden death is inadequate and that new approaches to this problem are needed. Further support for the hypothesis that complex ventricular arrhythmias after myocardial infarction are merely incidental and not critical to the subsequent development of sudden cardiac death comes from the recent Norwegian trial of timolol in patients recovering from an episode of acute myocardial infarction. ${ }^{8}$ In this study timolol was shown to significantly reduce the incidence of both sudden and nonsudden death after infarction. However, the reduction in incidence of sudden cardiac death by timolol was not reported to be associated with any significant reduction in complex ventricular ectopic activity.

If complex ventricular ectopic beats during the late hospital phase of acute myocardial infarction are merely "innocent bystanders" and markers of ventricular dysfunction and/or extensive ischemic heart disease rather than primary etiologic factors for subsequent sudden death, how can we explain the importance of ventricular dysfunction as a risk factor and what is its implication for the prevention of subsequent sudden cardiac death after infarction? Our current hypothesis, based upon our previous experience ${ }^{2,4,5}$ and that of others, is that extensive myocardial damage makes the ventricle vulnerable to what might otherwise be subthreshold stimuli. These stimuli may cause heterogeneous repolarization and electrical disorganization, resulting in ventricular fibrillation and sudden death. For example, a patient with extensive myocardial damage and multivessel coronary artery disease may develop ventricular fibrillation after administration of relatively small concentrations of catecholamines. Similar catecholamine concentrations in a patient wtih a normal ventricle without coronary artery disease would not cause ventricular fibrillation or sudden cardiac death. Thus, timolol could have prevented sudden cardiac death by blocking the effects of catecholamines in patients with extensive myocardial damage or coronary arterial narowing, or both. Other subthreshold humoral or neural humoral factors or both may also be of impotance and precipitate ventricular fibrillation in patients with extensive myocardial dysfunction. Recent studies in our laboratory suggest that intracerebral opiate administration can cause profound systemic and coronary vasoconstriction, ${ }^{8}$ and in the presence of 
extensive myocardial damage, it is possible that intracerebral endogenous opioid release could result in myocardial ischemia, ventricular fibrillation, and sudden cardiac death.

In the foregoing discussion it has been emphasized that left ventricular dysfunction and extensive coronary artery disease are important factors in the occurrence of sudden cardiac death. This, of course, is true only for those with ischemic heart disease. A relatively large percentage of patients who die suddenly do not have ischemic heart disease or ventricular dysfunction. Sudden cardiac death in these patients without known cardiac disease may be due to threshold rather than subthreshold stimuli. For example, a release of high concentrations of catecholamines and/or other neural or humoral factors may be required to cause inhomogeneity in ventricular conduction and to precipitate sudden death in the normal ventricle. The incidence of sudden death in those without myocardial damage and ischemic heart disease is relatively low since it is relatively uncommon that these neural or humoral factors reach threshold levels. On the other hand, in the patient with extensive myocardial dysfunction and ischemic heart disease, catecholamine levels in the coronary sinus are relatively high. The presence of left ventricular dysfunction and an increased vulnerability to ventricular fibrillation may in conjunction with other neural or humoral factors lead to sudden death. If this hypothesis is correct, the approach to the prophylaxis of sudden cardiac death will depend upon a better understanding of the neural and humoral stimuli, both subthreshold and threshold, which result in ventricular fibrillation. If these stimuli can be identified and the factors that control their release and mechanisms of action understood, it is possible that appropriate prophylactic strategies for the prevention of sudden cardiac death could be developed. Development of new antiarrhythmic agents, the use of extensive Holter electrocardiographic recording, and the application of electrophysiologic techniques may be of benefit to the patient with symptomatic ventricular tachycardia and/or recurrent ventricular fibrillation, but these techniques have not and probably will not be of benefit in developing an effective prophylactic approach to the overall problem of sudden cardiac death or to the particular subset of patients surviving an episode of acute myocardial infarction with extensive left ventricular dysfunction.

\section{REFERENCES}

1. Kotler, M. N., B. Tabatznik, M. M. Mower \& S. Tominaga. 1973. Prognostic significance of ventricular ectopic beats with respect to sudden death in the late postinfarction period. Circulation 47: 959-966.

2. Schulze, R. A., H. W. Strauss \& B. Pitt. 1977. Sudden death in the year following myocardial infarction. Relation to ventricular premature contractions in the late hospital phase and left ventricular ejection fraction. Am. J. Med. 62: 192-199.

3. Ruberman, W., E. Weinglatt, J. Goldberg, C. Frank \& S. Shapiro. 1977. Ventricular premature beats and mortality after myocardial infarction. $\mathbf{N}$. Engl. J. Med. 297: 750.

4. Schulze, K. A., J. O. Humphries, L. S. C. Griffith, H. Ducci, S. Achuff, M. G. Baird, E. D. Mellits \& B. Pitr. 1977. Left ventricular and coronary angiographic anatomy. Relationship to ventricular irritability in the late hospital phase of acute myocardial infarction. Circulation 55: 839-843.

5. Taylor, G. J., J. O. Humphries, E. D. Mellits, B. Pitt, R. A. Schulze, 
L. S. C. Griffith \& S. C. Achuff. 1980. Predictors of clinical course, coronary anatomy and left ventricular function after recovery from acute myocardial infarction. Circulation 62: 960-970.

6. Kleiger, R. E., J. P. Miller, S. Thanavaro, M. A. Province, T. F. Martin \& G. C. Olrver. 1981. Relationship between clinical features of acute myocardial infarction and ventricular runs 2 weeks to 1 year after infarction. Circulation 63: 64-70.

7. Chamberlain, D. A., D. E. Jewitt, D. G. Julian, R. W. F. Campbell, D. M. C. Boyle, R. G. Shanks, et al. 1980 . Oral-mexiletine in high-risk patients after myocardial infarction. Lancet 2: 8208-8209.

8. Norwegian Multicenter Study Group. 1981. Timolol-induced reduction in mortality and reinfarction in patients surviving acute myocardial infarction. N. Engl. J. Med. 304: 801-807.

9. Pasyk, S., J. A. Walton \& B. Pitt. 1981. Central opoiod mediated coronary and systemic vasoconstriction in the conscious dog. Circulation 64 (Suppl. IV): IV-41.

\section{Discussion}

R. Baigrie (Toronto, Ontario, Canada): Dr. Pitt could you tell me what percentage of sudden deaths in North America each year are postinfarction sudden deaths?

Dr. PItr: Perhaps Dr. Cobb would be better prepared to answer that.

Dr. CoBB: It is difficult to answer with certainty, but this question is very important. Of the patients that we see after resuscitation from out-of-hospital ventricular fibrillation due to coronary disease, $60 \%$ have no history of prior myocardial infarction, and $40 \%$ do. About $15 \%$ of the patients appear to have had a myocardial infarction within the preceding 6 months to a year. So while they do not represent an enormous part of the sudden death population, this subset is obviously very important.

DR. P. Gallerstein (New York, New York): We have noticed that in a lot of the patients coming to our coronary care unit with very poor left ventricular function and complex arrhythmias, the arrhythmias have actually subsided as we have sought to improve left ventricular function by the very aggressive use of both pre- and afterload agents. Have you looked at this?

Dr. PITT: We haven't examined this directly, but the few data that I am familiar with seem to indicate that although ventricular function can be altered by afterload-reducing agents, the overall mortality has not changed. Perhaps these premature ventricular contractions are really markers, but not necessarily the event that triggers death.

Dr. Herling: You appropriately negate the role of empirical antiarrhythmic therapy in preventing sudden death in the high-risk subgroup, but how do you regard the role of programmed electrical stimulation in the electrophysiology laboratory to identify those high-risk subsets who may sustain recurrent ventricular tachycardia?

DR. PItT: This will be discussed later by others. 